\title{
Répartition de l'entomofaune pollinisatrice sur des fleurs de colza (Brassica napus $L$ ) et de navette (Brassica campestris $L$ ) : incidence du caractère apétale de la navette
}

\author{
E Brunel 1, J Mesquida 1, M Reriard 2, X Tanguy 2 \\ 1 INRA, laboratoire de zoologie, \\ 2 INRA, station d'amélioration des plantes, centre de recherche de Rennes, \\ domaine de la Motte au Vicomte, BP 29, F 35650 Le Rheu, France
}

(Reçu le 16 décembre 1992; accepté le 11 mai 1993)

\begin{abstract}
Résumé - Disposant d'une variété de navette apétale, la composition et la répartition de la faune pollinisatrice locale ainsi que certains facteurs d'attractivité (densité de fleurs, sécrétions qualitatives et quantitatives du nectar) ont été comparés à ceux d'une navette à fleurs "normales" (ou témoin) et à ceux de 2 lignées de colza. La transformation de la morphologie florale chez la navette apétale n'a pas semblé s'accompagner d'une quelconque modification du comportement de butinage des insectes pollinisateurs. Des différences importantes sont cependant apparues entre les colzas et les navettes. Pour un même nombre d'insectes pollinisateurs observés sur les colzas et les navettes, les proportions d'Hyménoptères (Apis mellifera principalement) ont été significativement plus importantes sur les colzas. Sur les navettes, à l'inverse, ce sont les proportions de Diptères (Syrphidae notamment) qui ont dominé largement.
\end{abstract}

Apis mellifera / Syrphidae / butinage / plante apétale / sécrétion nectarifère / Brassica

\section{INTRODUCTION}

Le développement de la culture du colza et de la navette aussi bien en Europe qu'en Chine et en Amérique du Nord s'accompagne souvent d'une augmentation des problèmes sanitaires. Pour certaines maladies, le sélectionneur dispose d'une variabilité génétique importante dans l'espèce, lui permettant d'obtenir des lignées résistantes (nécrose du collet, cylindrosporiose, maladies des taches blanches et alternariose). Par contre, la sélection paraît moins efficace pour l'amélioration de la résistance à la sclérotiniose. La sélection de génotypes à fleurs sans pétales pourrait cependant conduire à l'obtention de variétés "résistantes" à cette maladie dans la mesure où la colonisation des pétales collés sur les feuilles est une étape nécessaire à la contamination des tissus sains (Brun et al, 1989). Il est donc envisagé de développer à l'avenir la culture de colzas et de navettes sans pétales. 
L'apparition de génotypes à fleurs dépourvues de pétales pourrait cependant poser des problèmes de pollinisation dans l'hypothèse où l'absence de pétales aurait une influence soit sur le comportement de butinage des insectes (répartition, attractivité des lignées vis-à-vis des insectes pollinisateurs), soit sur l'efficacité de la pollinisation, plus particulièrement dans l'éventualité de leur utilisation en production de semences hybrides F1.

C'est au cours de la phase initiale d'exploration des insectes que les facteurs d'attractivité (fleurs, couleur, arômes, nectar, etc) jouent un rôle important dans le comportement de butinage. C'est donc à ce moment là qu'une transformation de la morphologie florale peut entraîner une moins bonne attractivité vis-à-vis des insectes pollinisateurs, ce qui pourrait se traduire par une diminution de la productivité.

Ainsi, disposant d'une navette sans pétales, nous avons tenté d'établir une relation entre la morphologie de la fleur et sa fréquentation par les pollinisateurs en comparant cette navette apétale à une navette et à 2 lignées de colza à fleurs "normales».

\section{MATÉRIEL ET MÉTHODES}

Deux variétés de navette d'hiver (Brassica campestris L) ont été utilisées: I'une à fleurs sans pétales (mutation spontanée contrôlée par un gène récessif) obtenue par les établissements Lembke (Allemagne) et l'autre à fleurs "normales" ou témoin (variété "Chicon") ainsi que $2 \mathrm{li}$ gnées de colza d'hiver (Brassica napus L) "Hokkaiido" et "Samouraï" à fleurs "normales".

Cette étude a été effectuée en 1992 dans un dispositif expérimental mis en place au champ en 1991 sur le domaine de la Station d'amélioration des plantes de I'INRA du Rheu (Ille-etVilaine). II s'agit d'un dispositif en bloc complet équilibré à 4 variétés et 4 répétitions. La parcelle élémentaire était composée de 5 lignes de
$8 \mathrm{~m}$ de long avec des interlignes de $0,25 \mathrm{~m}$ (densité : 60 à 80 pieds au mètre carré). Les interparcelles étaient de $0,50 \mathrm{~m}$ et les interblocs de $2,50 \mathrm{~m}$.

Toutes les observations ont été effectuées à la $18^{e}$ et à la $19^{e}$ semaines, période qui correspond aux floraisons maximales des navettes et des colzas de l'essai. Elles ont porté sur des aspects phénologiques et entomologiques.

\section{Sur les aspects phénologiques}

II s'agissait d'estimer les densités de fleurs et les sécrétions nectarifères des différents génotypes. Les densités de fleurs ont été obtenues en effectuant plusieurs comptages des fleurs situées dans un espace matérialisé à l'aide d'un cerceau métallique de $50 \mathrm{~cm}$ de diamètre que l'on dispose au hasard sur le plateau de floraison de chaque parcelle (Taséi, 1978).

Les quantités de nectar sécrété par les fleurs ont été mesurées par la méthode du pipetage (micropipettes de $5 \mu$ ) décrite dans une note antérieure (Mesquida et al, 1988a) et appliquée à des fleurs de même âge prélevées sur 5 plantes. Les concentrations en matière sèche totale (ou sucres totaux), du nectar ont été obtenues à l'aide d'un réfractomètre de précision gradué de 0 à $90 \%$ (modèle Atago $n^{\circ} 8153$, Japon).

\section{Sur les aspects entomologiques}

Nous avons effectué l'inventaire des populations de butineuses sur chaque variété. Les insectes pollinisateurs ont été dénombrés sur une bande de $0,50 \mathrm{~m}$ de large et de $8 \mathrm{~m}$ de long sur chaque parcelle élémentaire. La largeur de la bande était matérialisée à l'aide d'un bâton de $0,50 \mathrm{~m}$ que l'on déplaçait tout au long de la parcelle selon la méthode décrite par Lecomte (1968).

Les dénombrements d'insectes ont été réalisés par beau temps au moment de l'activité maximale de la journée : le 27 avril $\left(17^{\mathrm{e}} \mathrm{sem}\right)$ à $12 \mathrm{~h}$ (HGMT) le 3 mai (18 sem) à $9 \mathrm{~h}$ et à $11 \mathrm{~h}$.

Les insectes ont été inventoriés dans la mesure du possible par espèce, par genre ou par groupe de familles dans les cas difficiles à déterminer à vue sur le terrain. Nous n'avons tenu compte que des insectes qui se trouvaient sur 
les fleurs, c'est-à-dire des insectes considérés comme étant des pollinisateurs potentiels.

Nous avons utilisé le test non paramétrique de Kruskal et Wallis (1952) pour analyser et comparer les populations d'insectes dénombrées sur chaque variété. Dans le cas du rejet de l'hypothèse nulle, les comparaisons de moyenne à $P=0,05$ ont été réalisées sur la somme des rangs (Hollander et Wolfe, 1973).

Les pourcentages de populations d'insectes pollinisateurs estimés sur chaque génotype ont été comparés au moyen du test $\chi^{2}$.

Les moyennes sont données avec l'erreurtype ( $s / \sqrt{ } n$ où $s$ est l'écart type).

\section{RÉSULTATS}

\section{Densités de fleurs}

Pendant la période d'observation des populations de butineuses (18 $\mathrm{sem})$, les densités de fleurs étaient en moyenne de 2200 fleurs au mètre carré pour la navette sans pétales, 1920 pour la navette (témoin), 1250 et 1600 respectivement pour "Hokkaïdo" et "Samouraï» soit des proportions de l'ordre de : $32,28,18$ et $22 \%$, qui ne sont pas significativement différentes d'une proportion théorique équivalente à $25 \%\left(\chi^{2}=4,64<P 0,20\right.$ à $\left.3 \mathrm{ddl}\right)$.

\section{Les sécrétions nectarifères}

Les moyennes par fleur des sécrétions nectarifères de la navette sans pétale $(0,72 \pm 0,25 \mu l)$ ont été équivalentes à celles de la navette témoin $(0,73 \pm 0,20$ $\mu \mathrm{m})$, mais celles des 2 variétés de colza ont été significativement plus élevées ( $P=$ $0,002)(1,8 \pm 0,75$ et $2,07 \pm 0,74 \mu \mathrm{l}$, soit de l'ordre de 2,5 à 3 fois plus). Les concentrations en matière sèche du nectar des 4 variétés ont été, par contre, identiques (de l'ordre de 23 à $31 \%$, différences non significatives).

\section{Faune pollinisatrice}

Sur un effectif total de 554 individus, la faune pollinisatrice était composée en moyenne de :

- 44\% d'abeilles domestiques (Apis mellifera) ;

$-22 \%$ d'abeilles solitaires, (diverses espèces d'Hyménoptères) ;

$-2 \%$ de bourdons (Bombus spp) ;

- 32\% de mouches (Diptères : Syrphidae, Anthomyiidae et Scatophagidae).

\section{Répartition des populations de butineuses}

La répartition des populations de butineuses (toutes espèces confondues) a été homogène sur toutes les variétés (différences non significatives) mais selon les espèces, le tableau I met en évidence :

1) des densités d'abeilles domestiques significativement plus élevées $(P=0,001$ et $0,02)$ sur les colzas que sur les navettes (de l'ordre de 3 à 7 fois plus, tableau I-1) et des proportions observées très nettement différentes ;

2) des populations d'abeilles solitaires et de bourdons réparties d'une façon relativement homogène sur toutes les variétés (tableau I-2) ;

3) une polarisation importante des populations de Diptères sur les navettes (de l'ordre de 2 à 7 fois plus par rapport aux colzas, tableau I-3) ;

4) aucune différence significative n'a été mise en évidence entre les 2 types de navettes (tableau I-1, $1-2,1-3$ ).

Les tests $\chi^{2}$ significatifs $(P=0,001) \mathrm{du}$ tableau II permettent de constater que finalement les abeilles domestiques sont proportionnellement beaucoup plus attirées 
Tableau I. Répartition des populations d'abeilles domestiques, d'abeilles solitaires + bourdons et de mouches par date et heure d'observations et par variété (moyennes et \% pour $4 \mathrm{~m}^{2}$ ).

\begin{tabular}{|c|c|c|c|c|c|c|}
\hline & & \multicolumn{4}{|c|}{ Variétés } & \multirow{2}{*}{$\underset{\chi^{2}}{\text { Signif }}$} \\
\hline & & $\mathrm{Na} a p$ & Na té & $\mathrm{Co} \mathrm{Ho}$ & Co Sa & \\
\hline \multicolumn{7}{|c|}{ 1-1 Abeilles domestiques } \\
\hline $\begin{array}{l}27 \text { avril } \\
\text { à } 12 \mathrm{~h} \\
03 \text { mai } \\
\text { à } 9 \mathrm{~h} \\
03 \text { mai } \\
\text { à } 11 \mathrm{~h}\end{array}$ & $\begin{array}{l}\text { Moy } \\
(\%) \\
\text { Moy } \\
(\%) \\
\text { Moy } \\
(\%)\end{array}$ & $\begin{array}{l}1,5( \pm 1.0) \\
(11 \%) \mathrm{A} \\
1,25( \pm 0,5) \mathrm{A} \\
(7 \%) \mathrm{A} \\
3,75( \pm 2,1) \mathrm{A} \\
(12 \%) \mathrm{A}\end{array}$ & $\begin{array}{l}2,25( \pm 1,3) \\
(16 \%) A \\
1,0( \pm 0,8) A \\
(5 \%) A \\
3,5( \pm 1,7) A \\
(12 \%) A\end{array}$ & $\begin{array}{l}5,50( \pm 6,5) \\
(40 \%) \mathrm{B} \\
7,5( \pm 1,9) \mathrm{B} \\
(41 \%) \mathrm{B} \\
11,0( \pm 1,8) \mathrm{B} \\
(36 \%) \mathrm{B}\end{array}$ & $\begin{array}{l}4,50( \pm 2.9) \\
(33 \%) \mathrm{B} \\
8,5( \pm 2.4) \mathrm{B} \\
(47 \%) \mathrm{B} \\
12,0( \pm 2.6) \mathrm{B} \\
(40 \%) \mathrm{B}\end{array}$ & $\begin{array}{c}4,2 \mathrm{NS}(1) \\
33,5 \mathrm{~S}^{\star \star \star}(2) \\
11,4 \mathrm{~S}^{\star \star}(1) \\
59,0 \mathrm{~S}^{\star \star \star}(2) \\
11,4 \mathrm{~S}^{\star \star}(1) \\
27,0 \mathrm{~S}^{\star * *}(2)\end{array}$ \\
\hline \multicolumn{7}{|c|}{ 1-2 Abeilles solitaires + bourdons } \\
\hline $\begin{array}{l}27 \text { avril } \\
\text { à } 12 \mathrm{~h} \\
03 \text { mai } \\
\text { à } 9 \mathrm{~h} \\
03 \text { mai } \\
\text { à } 11 \mathrm{~h}\end{array}$ & $\begin{array}{l}\text { Moy } \\
(\%) \\
\text { Moy } \\
(\%) \\
\text { Moy } \\
(\%)\end{array}$ & $\begin{array}{l}2,5( \pm 1.3) \\
(34 \%) \\
2,25( \pm 0,5) \\
(21 \%) \\
4,0( \pm 2.2) \\
(27 \%)\end{array}$ & $\begin{array}{l}2,0( \pm 1.2) \\
(28 \%) \\
3,0( \pm 2.7) \\
(28 \%) \\
5,0( \pm 2.9) \\
(30 \%)\end{array}$ & $\begin{array}{l}1,25( \pm 1.0) \\
(17 \%) \\
2,75( \pm 1.5) \\
(26 \%) \\
3,25( \pm 1.0) \\
(22 \%)\end{array}$ & $\begin{array}{l}1,5( \pm 1.9) \\
(21 \%) \\
2,75( \pm 1.5) \\
(25 \%) \\
2,75( \pm 0.96) \\
(18 \%)\end{array}$ & $\begin{array}{l}2,0 \text { NS (1) } \\
6,8 \text { NS (2) } \\
0,2 \text { NS (1) } \\
1,0 \text { NS (2) } \\
2,2 \text { NS (1) } \\
3,4 \text { NS (2) }\end{array}$ \\
\hline \multicolumn{7}{|c|}{ I-3 Mouches } \\
\hline $\begin{array}{l}27 \text { avril } \\
\text { à } 12 \mathrm{~h} \\
03 \text { mai } \\
\text { à } 9 \mathrm{~h} \\
03 \text { mai } \\
\text { à } 11 \mathrm{~h}\end{array}$ & $\begin{array}{l}\text { Moy } \\
(\%) \\
\text { Moy } \\
(\%) \\
\text { Moy } \\
(\%)\end{array}$ & $\begin{array}{l}5,25( \pm 2,9) \\
(32 \%) B \\
3,5( \pm 1, .7) A B \\
(31 \%) B \\
4,0( \pm 0,8) A B \\
(25 \%) A B\end{array}$ & $\begin{array}{l}5,25( \pm 4,6) \\
(32 \%) \mathrm{B} \\
5,75( \pm 1,7) \mathrm{B} \\
(51 \%) \mathrm{B} \\
6,75( \pm 0,9) \mathrm{B} \\
(42 \%) \mathrm{B}\end{array}$ & $\begin{array}{l}3,25( \pm 1,0) \\
(20 \%) \mathrm{A} \\
0,75( \pm 1,0) \mathrm{A} \\
(7 \%) \mathrm{A} \\
2,0( \pm 0) \mathrm{A} \\
(13 \%) \mathrm{A}\end{array}$ & $\begin{array}{l}2,50( \pm 0,6) \\
(16 \%) \mathrm{A} \\
1,25( \pm 0,5) \mathrm{A} \\
(11 \%) \mathrm{A} \\
3,25( \pm 1,5) \mathrm{AB} \\
(20 \%) \mathrm{AB}\end{array}$ & $\begin{array}{c}2,2 \mathrm{NS}(1) \\
8,2 \mathrm{~S}^{\star}(2) \\
10,0 \mathrm{~S}^{\star \star}(1) \\
49,0 \mathrm{~S}^{\star \star \star}(2) \\
11,3 \mathrm{~S}^{\star \star}(1) \\
18,0 \mathrm{~S}^{\star \star \star}(2)\end{array}$ \\
\hline
\end{tabular}

Na ap : navette apétale ; Na té : navette (témoin) ; Co Ho : colza var Hokkaïdo ; Co Sa : colza var Samouraï ; (1) : résultats de test $\chi^{2}$ à 3 ddl des valeurs moyennes; (2) : résultats du test $\chi^{2}$ à 3 ddl appliqué aux pourcentages de populations. Signif $=$ signification : NS $=$ différences non significatives à $P=0,05 ; S^{*}$ : différences significatives à $P=0,05 ; S^{\star \star}$ : différences significatives à $P=0,01 ; \mathrm{S}^{\star \star \star}$ : différences significatives à $P=0,001$. Les moyennes et les pourcentages suivis d'une même lettre ne sont pas significativement différents à $P=0,05$.

par les 2 lignées de colza (de l'ordre de 53 à $68 \%$ des populations totales contre 7 à $29 \%$ pour les Diptères), et inversement ce sont les Diptères qui le sont sur les 2 variétés de navettes (50 à 59\% contre 10 à $22 \%$ pour les abeilles). Pour chaque espèce on ne fait apparaître aucune différence entre variétés.

\section{DISCUSSION ET CONCLUSION}

Les comparaisons des fréquentations des insectes vis-à-vis des plantes apétales ont été réalisées dans un essai comprenant des plantes qui présentaient une bonne synchronisation de leur floraison. Les observations réalisées volontairement au 
Tableau II. Répartition (en \%) des populations de butineuses par groupe d'insectes, par date et heure d'observation et par variété.

\begin{tabular}{|c|c|c|c|c|c|c|}
\hline & & \multicolumn{4}{|c|}{ Variétés } & \multirow{2}{*}{$\begin{array}{r}\text { Signif } \\
x^{21}\end{array}$} \\
\hline & & Na ap & Na té & $\mathrm{CoHO}$ & Co Sa & \\
\hline 27 avril & $A b$ do & $14 \%$ & $22 \%$ & $55 \%$ & $53 \%$ & \\
\hline \multirow[t]{2}{*}{ à $12 \mathrm{~h}$} & $\begin{array}{l}A b \text { so } \\
B o\end{array}$ & $\begin{array}{r}30 \% \\
0 \%\end{array}$ & $\begin{array}{r}22 \% \\
0 \%\end{array}$ & $\begin{array}{c}12 \% \\
0 \%\end{array}$ & $\begin{array}{r}18 \% \\
0 \%\end{array}$ & \\
\hline & Mo & $56 \%$ & $56 \%$ & $33 \%$ & $29 \%$ & 59,8 \\
\hline \multicolumn{2}{|c|}{ Total } & $100 \%$ & $100 \%$ & $100 \%$ & $100 \%$ & $\mathrm{~S}^{\star \star *}$ \\
\hline \multicolumn{2}{|c|}{ Effectif total : 148} & 37 & 37 & 40 & 34 & \\
\hline 3 mai & $A b$ do & $18 \%$ & $10 \%$ & $68 \%$ & $68 \%$ & \\
\hline \multirow[t]{3}{*}{ à $9 \mathrm{~h}$} & Ab so & $29 \%$ & $26 \%$ & $20 \%$ & $18 \%$ & \\
\hline & Bo & $4 \%$ & $5 \%$ & $5 \%$ & $4 \%$ & \\
\hline \multirow{2}{*}{\multicolumn{2}{|c|}{ Total }} & $50 \%$ & $59 \%$ & $7 \%$ & $10 \%$ & 145 \\
\hline & & $100 \%$ & $100 \%$ & $100 \%$ & $100 \%$ & $S^{\star \star \star}$ \\
\hline \multicolumn{2}{|c|}{ Effectif total : 161} & 28 & 39 & 44 & 50 & \\
\hline $3 \mathrm{mai}$ & $A b$ do & $32 \%$ & $23 \%$ & $68 \%$ & $67 \%$ & \\
\hline \multirow[t]{3}{*}{ à $11 \mathrm{~h}$} & Ab so & $30 \%$ & $33 \%$ & $12 \%$ & $14 \%$ & \\
\hline & Bo & $4 \%$ & $0 \%$ & $8 \%$ & $1 \%$ & 85 \\
\hline & Mo & $34 \%$ & $44 \%$ & $12 \%$ & $18 \%$ & $S^{\star * *}$ \\
\hline \multicolumn{2}{|c|}{ Total } & $100 \%$ & $100 \%$ & $100 \%$ & $100 \%$ & \\
\hline \multicolumn{2}{|c|}{ Effectif total : 245} & 47 & 61 & 65 & 72 & \\
\hline
\end{tabular}

Na ap : navette apétale ; Na té : navette (témoin) ; Co Ho : colza var Hokkaïdo ; Co Sa : colza var Samouraï; ${ }^{1} \chi^{2}$ à $9 \mathrm{ddl}, \mathrm{Ho}=$ indépendance entre pollinisateurs et variétés. Ab do : abeilles domestiques (Apis mellifera); Ab so abeilles solitaires (toutes espèces confondues); Bo: bourdons (toutes espèces confondues); Mo: mouches (toutes espèces confondues).

maximum de la floraison des colzas et des navettes et en période de forte activité des insectes pollinisateurs (entre $9 \mathrm{~h}$ et $12 \mathrm{~h}$ ), ont permis de dégager un certain nombre de remarques.

Les fleurs de colzas et des navettes ont été très activement visitées par des espèces très diverses appartenant à l'ordre des Hyménoptères (abeilles domestiques, $A$ mellifera; diverses espèces d'abeilles solitaires et de bourdons, Bombus spp) et à celui des Diptères (Anthomyiidae, Syrphidae et Scatophagidae).
En général, la notion de pollinisateur s'applique essentiellement aux abeilles, aux bourdons et aux Diptères. Récemment, au moyen de pièges installés dans un dispositif de production de semences hybrides de colza, Brunel et al (1989) ont montré que les gros insectes capturés ne sont qu'une minorité. Parmis les Diptères, les Syrphidae et les Calliphoridae sont les plus remarquables car ils sont présents dans tous les biotopes à la recherche de protéines (pollen) et de matières énergétiques (nectar) nécessaires à leur ovogenèse. 
Les Calliphoridae sont d'ailleurs couramment utilisés en amélioration des plantes pour effectuer des croisements entre lignées de colza car ils sont non nuisibles et faciles à élever. Les syrphes ont donné lieu à quelques études dans le domaine de la pollinisation : capacité à transporter du pollen sur leur corps (Simic et Radisic, 1990), présence dans des dispositifs en bandes alternées pour la production de semences F1 chez le colza (Brunel et al, 1989).

La répartition des insectes totaux sur les colzas et sur les navettes est relativement homogène. Antérieurement ou postérieurement à la phase de pleine floraison, les variations de densité de fleurs entre variétés et entre espèces auraient eu probablement une forte incidence sur la répartition des insectes. On sait, par exemple, qu'au cours de la très longue période de la floraison des colzas (de 4 à 6 semaines selon les années) les densités de populations de butineuses (abeilles domestiques) varient corrélativement avec l'intensité de la floraison (Taséi, 1978; Houedry, communication personnelle).

D'importantes différences apparaissent lorsque l'on s'intéresse plus particulièrement à la répartition spécifique des différents insectes selon les espèces.

Chez les colzas, on constate que la faune pollinisatrice est constituée de près de $90 \%$ d'Hyménoptères avec une très forte dominance d'abeilles domestiques, de nombreuses abeilles solitaires et quelques bourdons; les Diptères ne constituent qu'une très faible partie de la faune pollinisatrice du colza ( $10 \%$ environ); ils sont représentés par des espèces du groupes des Scatophagidae, des Syrphidae et des Anthomyiidae; ce même type de répartition a déjà été observé dans d'autres essais sur le colza (Mesquida et al, 1988/b).

Chez les navettes, on assiste à une inversion des proportions d'insectes: les abeilles, plus attirées par les colzas que par les navettes, sont remplacées par les Diptères (Scatophagidae, Syrphidae et Anthomyiidae); les abeilles solitaires sont aussi nombreuses que les abeilles domestiques et les bourdons restent toujours faiblement représentés.

La navette apétale s'est montrée aussi attractive que la navette avec pétales (témoin) tant au niveau des insectes totaux qu'au niveau des abeilles domestiques, des abeilles solitaires et des Diptères. Elle s'est en outre montrée aussi attractive que les 2 lignées de colza vis-à-vis des abeilles solitaires, mais elle a été significativement moins attractive vis-à-vis des abeilles domestiques et plus vis-à-vis des Diptères.

Les 2 lignées de colza n'ont fait apparaître aucune différence entre elles. C'est donc entre espèces que les différences existent.

Les colzas attirent plus d'abeilles domestiques que les navettes, ce qui peut s'expliquer par les différences quantitatives de nectar sécrété par fleur; des travaux ont déjà démontré l'importance des sécrétions nectarifères qualitatives et quantitatives sur le comportement de butinage des abeilles (Beutler, 1953; Baker et Baker, 1975 ; Heinrich, 1975; Masson, 1983; Mesquida et al, 1988b).

Par contre, aucune explication satisfaisante ne peut être avancée concernant la forte attractivité des navettes vis-à-vis des Diptères. On peut cependant formuler plusieurs hypothèses qu'il resterait à vérifier. La forte attractivité des navettes vis-à-vis des Diptères serait liée :

- aux constituants chimiques de la plante tels que des glucosinolates ou aux substances volatiles (arômes) émises par la plante; - aux constituants glucidiques des nectars; en ce qui concerne le colza, de nombreuses études ont déjà été effectuées (Mesquida et al, 1988a, 1991), par contre, la composition glucidique du nectar des navettes reste à préciser. 
En conclusion, on peut dire que dans les conditions de notre expérimentation la transformation de la morphologie florale chez la navette ne s'est pas traduite par une modification de l'attractivité vis-à-vis des populations de butineuses totales constituées d'Hyménoptères et de Diptères.

II serait intéressant de déterminer les facteurs d'attractivité qui ont permis de différencier la navette du colza de façon à inclure éventuellement ces critères dans des programmes de création variétale en vue d'une optimisation du comportement de butinage de la faune pollinisatrice présente dans le milieu.

\section{Summary - Distribution of pollinating entomofauna on rapeseed (Brassica na-} pus $\mathrm{L}$ ) and turnip rape (Brassica campestris $L$ ) flowers: the effect of the turnip rape's apetalous character. Foraging behavior and spatial distribution of insect pollinators depend on numerous parameters such as attractivity factors (nectar, flowers, color, aroma). Morphological modifications in flowers could induce changes in attractiveness to pollinators. An apetalous turnip rape was compared to rapeseed (var Hokkaïdo and Samouraï) and also turnip rape (var Chicon) varieties with welldeveloped petals. Comparisons were carried out on flowering and pollinating entomofauna. A $1.5 \times 8.0-\mathrm{m}$ plot size was used in a randomized complete block design with 4 replicates and 4 varieties.

At full flowering and at optimal insect activity, various parameters were estimated: flower density (flower number per $\mathrm{m}^{2}$ ), nectar production per flower (pipetting method), sugar concentration (refractometer) and pollinator insect distribution (insect number on $0.5 \times 0.8 \mathrm{~m}$ strips for each, plant variety and for each block).

The results demonstrated that: 1) flower densities on rapeseed and turnip rape were equal; 2) nectar secretion was quantitatively and qualitatively similar in turnip rape varieties but quantitatively less when compared to rapeseed; 3 ) the pollinators on rapeseed and turnip rape varieties were mainly composed of Hymenoptera (in particular A mellifera) and Diptera; 4) the total number of pollinator insects was the same on rapeseed and turnip rape varieties; 5) honeybee density was significantly higher (3 to 7-fold higher; table 1-1) on turnip rape than on rapeseed; 6 ) the distribution of other Hymenoptera (ie solitary bees and bumblebees) did not differ between rapeseed and turnip rape varieties (table I-2); 7) Diptera density was significantly higher on turnip rape than on rapeseed (2 to 7 -fold higher; table I-3); 8) for the same total number of insects, the entomofauna was mainly composed of Hymenoptera (honeybees) on rapeseed and Diptera (Syrphidae) on turnip rape (table II); 9) apetalous turnip rape was as attractive to pollinators as the variety with petals; the results were the same for rapeseed varieties, betweenspecies differences were significant. In conclusion, it was found that lack of petals in turnip rape did not seem to affect the foraging behavior of local pollinating insects.

\section{Apis mellifera / Syrphidae / foraging / behavior / apetalous plant / nectar secretion / Brassica}

\section{Zusammenfassung - Verteilung der In-} sektenbestäuber auf den Blüten des Raps (Brassica napus L) und des Rübsen (Brassica campestris L): Vorkommen des Merkmals "apetal" (="blütenblätterlos") beim Rübsen. Das Sammelverhalten und die Verteilung der Blütenbesucher hängen von zahlreichen biotischen und abiotischen Faktoren $a b$, $\mathrm{zB}$ von den Faktoren der Attraktivität (Nektar, Blüten, Farbe, Aroma, usw). Eine Veränderung der Blütenmorphologie 
könnte also zu einer Veränderung der Attraktivität gegenüber den Bestäubern führen. Da wir über eine apetale Form des Rübsen verfügten, wollten wir diese Hypothese durch Vergleich des Blühablaufs und des Verhaltens der Bestäuber dieser apetalen Varietät prüfen, wobei wir als Kontrolle die Rübsensorte "Chicon" mit "normalen" Blüten als Kontrolle verwendeten, zusammen mit zwei Rapslininien ("Hokkaido" und "Samurai").

Die Versuche wurden in Blockanordnung zu 4 Varietäten und 4 Wiederholungen angelegt. Die Grundparzelle war 1.5 $\mathrm{m}$ breit und aus 5 Linien von $8 \mathrm{~m}$ Länge zusammengesetzt. Die Beobachtungen wurden zur Zeit des Höhepunkts der Blüte von Raps und Rübsen, zugleich mit voller Aktivität der bestäubenden Insekten, durchgeführt ; sie erstreckten sich auf die Dichte der Blüten (Blütenzahl pro $\mathrm{m}^{2}$ ), die Nektarmenge pro Blüte (Methode der Pipettierung), die Zuckerkonzentration (Messung mit Refraktometer) und die Verteilung der bestäubenden Insekten (Anzahl der Insekten auf Streifen von $8.0 \times 0.5 \mathrm{~m}$ pro Varietät und pro Block). ben :

Die Resultate haben folgendes erge-

1. Raps und Rübsen haben eine gleichgroBe Blühintensität

2. Die Nektarabsonderung des apetalen Rübsen war in Quantität und Qualität dieselbe wie bei der Kontrolle (Rübsen), aber von deutlich niedrigerer Quantität als beim Raps.

3. Im ganzen bestand die bestäubende Insektenfauna am Rübsen wie am Raps vorwiegend aus Hymenopteren (besonders Honigbienen) und zahlreichen Dipteren.

4. Die Gesamtzahl bestäubender Insekten war am Raps und am Rübsen gleich.

5. Die Dichte der Honigbienen war am Raps signifikant höher als am Rübsen (3$7 x$ so hoch, Tabelle (-1).
6. Die übrigen Hymenopteren (Solitärbienen, Hummeln) waren gleichmäßig über Raps und Rübsen verteilt (Tabelle I-2).

7. Die Dichte der Dipteren war am Rübsen signifikant höher als am Raps $(2-7 x$ so hoch, Tabelle I-3).

8.-Bei derselben Zahl beobachteter Insekten dominierten beim Raps sehr stark die Hymenopteren (besonders Honigbienen), hingegen beim Rübsen Dipteren (vorwiegend Syrphiden).

9. Die apetale Form des Rübsen zeigte hinsichtlich des Besuchs bestäubender Insekten keinen Unterschied gegenüber der Form mit "normalen" Blüten. Dasselbe war bei dem Vergleich der beiden Rapssorten der Fall. Die signifikanten Unterschiede liegen hingegen zwischen den beiden Arten.

Durch die Umbildung der Blütenmorphologie wird das Verhalten der besuchenden Insekten also nicht verändert.

\section{Apis mellifera / Syrphidae / Sammelver- halten / apetale Blüte / Brassica}

\section{RÉFÉRENCES}

Baker HG, Baker I (1975) Studies of nectar constitution and pollinator-plant coevolution. In: Coevolution of Animals and Plants (Le G Raven PH, eds) Proc Symp 5 1st int Cong Syst Evolution Biol Boulder, CO, 100-104

Beutler R (1953) Nectar. Bee World 34, 107-162

Brun $\mathrm{H}$, Renard M, Tribodet M, Plessis J, Tanguy $X$ (1989) Apport de la lutte génétique contre les maladies du colza. Phytoma 404, 36-41

Brunel E, Grootaert P, Mesquida J (1989) Entomofaune associée à la floraison du colza (Brassica napus $L$ ) : note préliminaire sur les Dolichopodidae et les Empididae (Insectes: Diptera). Med Fac Landbouww Rijksuniv Gent 54/3 a,727-737

Heinrich B (1975) Energetics of pollination. Annu Rev Ecol Syst 6, 139-170 
Hollander M, Wolfe DA (1973) Non-parametric Statistical Methods. John Wiley et Sons, New York

Kruskal WH, Wallis WA (1952) Use of ranks in one-criteron variance analysis. J Am Stat Assoc 47, 583-621

Lecomte J (1968) Le comportement de butinage. In : Traité de biologie de l'abeille. Masson, Paris t2, 402-415

Masson C (1983) Rôle des médiateurs chimiques d'origine animale et végétale dans la pollinisation. CR $5^{e}$ Symp Int Pollinisation. INRA éd Versailles, 25-37

Mesquida J, Marilleau R, Pham-Delegue MH, Renard M (1988/a) A study of rapeseed Brassica napus $L$ var oleifera Metzger) flower nectar secretions. Apidologie 19, 307-318
Mesquida J, Renard M, Pellan-Delourme R, Pelletier G, Morice J (1988b) Influence des sécrétions nectarifères des lignées mâlestériles pour la production de semences hybrides $\mathrm{F} 1$ de colza. In : Variabilité génétique cytoplasmique et stérilités mâle cytoplasmique. Colloq INRA, n 45, 269-280

Mesquida J, Pham-Delegue MH, Marilleau R, Le Metayer M, Renard M (1991) La sécrétion nectarifère des fleurs de cybrides mâlesstériles de colza d'hiver (Brassica napus L). Agronomie 11, 217-227

Simic S, Radisic P (1990) Hoverflies (Dipera Syrphidae) and pollen transport. CR 2nd int Congr Dipterology. Bratislava (publication affichée)

Taséi JN (1978) La floraison du colza et son butinage par les abeilles. Inf Tech CETIOM 60, $11-20$ 